\title{
ON 2-KNOT GROUPS WITH ABELIAN COMMUTATOR SUBGROUPS
}

\author{
KATSUYUKI YOSHIKAWA
}

\begin{abstract}
In this paper it is shown that if the commutator subgroup of a 2-knot group is abelian, then it is isomorphic to $Z \oplus Z \oplus Z, Z_{\alpha}, Z[1 / 2]$ or $Z[1 / 2] \oplus Z_{5}$, where $\alpha$ is an odd integer and $Z[1 / 2]$ is the additive group of the dyadic rationals.
\end{abstract}

1. Introduction. An $n$-knot $K$ is a smooth submanifold of a homotopy $(n+2)$ sphere $\Sigma^{n+2}$ which is homeomorphic to the $n$-sphere $S^{n}$. The fundamental group of the complement $\Sigma^{n+2}-K$ is called the group of $K$.

In [11], Levine completely determined 2-knot groups with finitely generated abelian commutator subgroups. He showed that a finitely generated abelian group is isomorphic to the commutator subgroup of a 2-knot group if and only if it is $Z \oplus Z \oplus Z$ or $Z_{\alpha}$, where $\alpha$ is an odd integer. In this paper, we will consider 2-knot groups whose commutator subgroups are abelian and not finitely generated, and the following result will be obtained.

THEOREM. Let $G$ be a 2-knot group. If the commutator subgroup $G^{\prime}$ is abelian and not finitely generated, then it is isomorphic to $Z[1 / 2]$ or $Z[1 / 2] \oplus Z_{5}$, where $Z[1 / 2]$ is the additive group of the dyadic rationals.

There exists a 2-knot group whose commutator subgroup is isomorphic to $Z[1 / 2]$ [8]. On the other hand, it still remains open whether $Z[1 / 2] \oplus Z_{5}$ can be realized as the commutator subgroup of a 2-knot group. If there exists such a 2-knot, its group would be presented by

$$
\left\langle a, b, t: t^{-1} a t=a^{2}, t^{-1} b t=b^{-1}, b^{5},[a, b]\right\rangle .
$$

Question. Does there exist a 2-knot group whose commutator subgroup is isomorphic to $Z[1 / 2] \oplus Z_{5}$ ?

2. Preliminaries. Let $X$ be an abelian group. We denote the torsion part of $X$ by $T(X)$ and denote $X / T(X)$ by $F(X)$. By the torsion-free rank $r(X)$ of $X$, we mean the dimension of the vector space $X \otimes Q$, where $Q$ is the field of rational numbers. Similarly, for a prime $p$, the $p$-rank $r_{p}(X)$ of $X$ is defined by the dimension of the vector space $T(X) \otimes F_{p}$ over $F_{p}$, where $F_{p}$ is the field of order $p$.

Received by the editors February 21, 1983.

1980 Mathematics Subject Classification. Primary 57Q45.

Key words and phrases. 2-knot, abelian commutator subgroup.

(C)1984 American Mathematical Society $0002-9939 / 84 \$ 1.00+\$ .25$ per page 
The second exterior power $\wedge^{2} X$ of $X$ is defined by $X \otimes X / D$, where $D$ $=\langle x \otimes x: x \in X\rangle$ [1]. We will denote an element $x_{1} \otimes x_{2}+D$ of $\wedge^{2} X$ by $x_{1} \wedge x_{2}$.

Let $1 \rightarrow R \rightarrow F \rightarrow X \rightarrow 1$ be a presentation of $X$. By [7], the second homology group $H_{2}(X)$ of $X$ is isomorphic to $R \cap[F, F] /[R, F]$. Since $X$ is abelian, we have $[F, F] \subset R$, and so $H_{2}(X) \cong[F, F] /[R, F]$. Therefore, $\wedge^{2} X$ and $H_{2}(X)$ are isomorphic by the mapping $f\left(x_{1} \wedge x_{2}\right)=\left[\tilde{x}_{1}, \tilde{x}_{2}\right][R, F]$, where $\tilde{x}_{1}, \tilde{x}_{2} \in F$ represent $x_{1}, x_{2} \in X$, respectively [16].

For an $n$-knot $K$, let $G$ be the group of $K$ and $\tilde{E}$ the infinite cyclic covering of the complement $\Sigma^{n+2}-K$. Then the homologies $H_{q}(\tilde{E})$ are left modules over the ring $\Lambda=Z\left[t, t^{-1}\right]$, where $t$ is a generator of the group of covering transformations [10]. Since $\pi_{1}(\tilde{E}) \cong G^{\prime}$, these $\Lambda$-module structures induce those on the integral homology groups $H_{q}\left(G^{\prime}\right), q=1,2$. More directly, we may consider that $H_{1}(G)$ operates on $H_{q}\left(G^{\prime}\right)$ by conjugation [11]. (This is well defined since inner automorphisms of $G^{\prime}$ induce the identity on homology.) If $G^{\prime}$ is abelian, then the left $\Lambda$-module structure on $\wedge^{2} G^{\prime}$ is given by $t\left(g_{1} \wedge g_{2}\right)=t g_{1} \wedge t g_{2}$ for any elements $g_{1}$ and $g_{2}$ of $G^{\prime}$.

In this paper, we will consider only modules over the commutative ring $\Lambda$. Thus we may not distinguish between left and right $\Lambda$-modules.

Let $H=\langle S: R\rangle$ be a group, and let $A$ and $B$ be subgroups of $H$ with an isomorphism $\phi: A \rightarrow B$. The $H N N$ extension of $H$ relative to $A, B$, and $\phi[6,12]$ is defined by the group

$$
G=\left\langle S, t: R, t^{-1} a t=\phi(a), a \in A\right\rangle .
$$

The group $H$ is called the base of $G$, and $A$ and $B$ are called the associated subgroups. We denote the group $G$ by $\operatorname{HNN}(H, A, B, \phi)$. We can see that the obvious homomorphism of $H$ to $G$ is a monomorphism [12]. Hence, we can consider $H$ as a subgroup of $G$.

From [4], we can easily see that any $n$-knot group $(n \geqslant 1)$ has a finitely presented base and finitely generated associated subgroups.

3. Lemmas. Throughout this section, let $G$ be a 2 -knot group with abelian commutator subgroup $G^{\prime}$. From $\S 2, G$ has a finitely presented base $H$. Since $H$ is contained in $G^{\prime}$, it is a finitely generated abelian group.

Proposition 3.1. Let $G=\operatorname{HNN}(H, A, B, \phi)$. Then $H=A$ or $H=B$.

Proof. Let $M$ be the subgroup of $G$ generated by elements $\left\{h, t^{-1} h t: h \in H\right\}$. Then $M$ can be considered as a free product of $t^{-1} H t$ and $H$ with amalgamated subgroups $t^{-1} A t$ and $B$. Since $M$ is contained in $G^{\prime}, M$ is abelian. Hence $M$ is indecomposable with respect to amalgamated product [13, p. 227]. Thus, we have $t^{-1} H t=t^{-1} A t$ or $H=B$. The proof is completed.

It is easily seen that $G^{\prime}$ is finitely generated if and only if $H=A=B$. From now on, we assume that $G^{\prime}$ is not finitely generated. Furthermore, without loss of generalities, we may assume that $H=A$ and $H \neq B$. Then $G^{\prime}$ is a union of an ascending chain of subgroups

$$
H<t H t^{-1}<t^{2} H t^{-2}<\cdots,
$$


and the torsion-free rank of $G^{\prime}$ is equal to the torsion-free rank of $H$. A $\Lambda$-module presentation of $G^{\prime}$ is given by

(1)

$$
\begin{aligned}
\left\langle a_{1}, \ldots, a_{n}, b_{1}, \ldots, b_{m}: t a_{i}\right. & =\sum_{j=1}^{n} \alpha_{i j} a_{j}+\sum_{j=1}^{m} \beta_{i j} b_{j}, \\
i & \left.=1, \ldots, n, t b_{k}=\sum_{j=1}^{m} \gamma_{k j} b_{j}, \lambda_{k} b_{k}=0, k=1, \ldots, m\right\rangle,
\end{aligned}
$$

where $n=r\left(G^{\prime}\right) ; \alpha_{i j}, \beta_{i j}, \gamma_{k j}, \lambda_{k} \in Z$; and $\lambda_{k} \neq 0$.

Since $\phi(T(H)) \subset T(B) \subset T(H)$, and $T(H)$ is finite, we have $T(B)=T(H)$. Therefore we see that $F(H) \supsetneqq F(B)$. Thus we get $\operatorname{det}\left(\alpha_{i j}\right) \neq \pm 1,0$. Furthermore, since $T\left(G^{\prime}\right)=T(H)$ is finite, it follows from [3, Proposition 100.2] that $G^{\prime}$ splits as an abelian group, i.e., $G^{\prime} \cong F\left(G^{\prime}\right) \oplus T\left(G^{\prime}\right)$.

Proposition 3.2. $F\left(G^{\prime}\right)$ and $T\left(G^{\prime}\right)$ are isomorphic to commutator subgroups of certain $n$-knot groups $(n \geqslant 3)$.

Proof. See [5 or 11].

For a $\Lambda$-module $M, \bar{M}$ denotes the conjugate module of $M$. That is, $\bar{M}$ is additively isomorphic to $M$, but $t$ acts on $\bar{M}$ identically with $t^{-1}$ on $M$. Levine proved the following

LemMA $3.3\left[10\right.$, p. $10 ; 11$, p. 251]. The second homology group $\mathrm{H}_{2}\left(G^{\prime}\right)$ is a $\Lambda$-homomorphic image of $\operatorname{Ext}_{\Lambda}^{1}\left(\overline{G^{\prime}}, \Lambda\right)$.

From definition of $F\left(G^{\prime}\right)$ and the presentation (1) of $G^{\prime}$, we have a $\Lambda$-module presentation of $F\left(G^{\prime}\right)$

$$
\left\langle a_{1}, \ldots, a_{n}: t a_{i}=\sum_{j=1}^{n} \alpha_{i j} a_{j}, i=1, \ldots, n\right\rangle .
$$

Let $\tilde{F}\left(G^{\prime}\right)$ be the $\Lambda$-module presented by

$$
\left\langle a_{1}, \ldots, a_{n}: t a_{i}=\sum_{j=1}^{n} \alpha_{j i} a_{j}, i=1, \ldots, n\right\rangle .
$$

Then we will show the following

LEMMA 3.4. $\operatorname{Ext}_{\Lambda}^{1}\left(\overline{G^{\prime}}, \Lambda\right)$ is $\Lambda$-isomorphic to $\overline{\tilde{F}\left(G^{\prime}\right)}$.

Proof. From (2), a free resolution of $F\left(G^{\prime}\right)$ as a $\Delta$-module is given by

$$
0 \rightarrow A_{n} \stackrel{t-\phi}{\rightarrow} A_{n} \rightarrow F\left(G^{\prime}\right) \rightarrow 0
$$

where $A_{n}$ is the free $\Lambda$-module with a basis $\left\{a_{1}, \ldots, a_{n}\right\}$, and $\phi$ is the $\Lambda$-endomorphism of $A_{n}$ defined by

$$
\phi\left(a_{i}\right)=\sum_{j=1}^{n} \alpha_{i j} a_{j}, \quad i=1, \ldots, n .
$$

Taking the dual $\operatorname{Hom}_{\Lambda}(\cdot, \Lambda)$ of this sequence, from [14, p. 60 and 10, p. 3] we 
obtain the exact sequence of $\Lambda$-modules

$$
0 \rightarrow \operatorname{Hom}_{\Lambda}\left(A_{n}, \Lambda\right) \stackrel{t-\phi^{*}}{\rightarrow} \operatorname{Hom}_{\Lambda}\left(A_{n}, \Lambda\right) \rightarrow \operatorname{Ext}_{\Lambda}^{1}\left(F\left(G^{\prime}\right), \Lambda\right) \rightarrow 0,
$$

where $\phi^{*}$ is the dual of $\phi$.

By [14], $\operatorname{Hom}_{\Lambda}\left(A_{n}, \Lambda\right)$ is the free $\Lambda$-module with a basis $\left\{h_{1}, \ldots, h_{n}\right\}$, where $h_{i}\left(a_{j}\right)=\delta_{i j}$ (the Kronecker symbol), $i, j=1, \ldots, n$. Therefore, a $\Lambda$-homomorphism $f$ of $A_{n}$ to $\operatorname{Hom}_{\Lambda}\left(A_{n}, \Lambda\right)$ given by $f\left(a_{i}\right)=h_{i}$ is a $\Lambda$-isomorphism. Under this isomorphism, $t-\phi^{*}$ induces the $\Lambda$-endomorphism $t-\tilde{\phi}$ of $A_{n}$, where $\tilde{\phi}\left(a_{i}\right)=$ $\sum_{j=1}^{n} \alpha_{j i} a_{j}, i=1, \ldots, n$ (cf. [1, p. 344]). Hence, we have the exact sequence of $\Lambda$-modules

$$
0 \rightarrow A_{n} \stackrel{t-\tilde{\phi}}{\rightarrow} A_{n} \rightarrow \operatorname{Ext}_{\Lambda}^{1}\left(F\left(G^{\prime}\right), \Lambda\right) \rightarrow 0
$$

Thus, $\operatorname{Ext}_{\Lambda}^{1}\left(F\left(G^{\prime}\right), \Lambda\right)$ is $\Lambda$-isomorphic to $\tilde{F}\left(G^{\prime}\right)$. Since

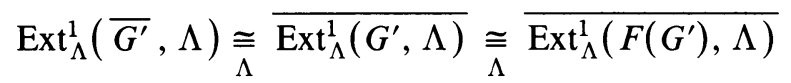

[10], this completes the proof.

REMARK. In a sense, Lemma 3.4 is a generalization of [11, p. 257].

LEMMA 3.5. The torsion-free rank of $H_{2}\left(F\left(G^{\prime}\right)\right)$ is equal to $n(n-1) / 2$, where $n=r\left(F\left(G^{\prime}\right)\right)$.

Proof. Let $k=n(n-1) / 2$. If $n=1$, then we have $\wedge^{2} F\left(G^{\prime}\right)=0$. For $n \geqslant 2$, let $\left(\hat{\alpha}_{i j}\right)_{1 \leqslant i, j \leqslant k}$ be the second exterior power of the matrix $\left(\alpha_{i j}\right)$ (see [1]). Then $\wedge^{2} F\left(G^{\prime}\right)$ is presented by

$$
\left\langle A_{1}, \ldots, A_{k}: t A_{i}=\sum_{j=1}^{k} \hat{\alpha}_{i j} A_{j}, i=1, \ldots, k\right\rangle .
$$

Therefore, the presentation matrix $M(t)$ of this presentation is $t I-\left(\hat{\alpha}_{i j}\right)$, where $I$ is the $k \times k$ unit matrix. Since $\operatorname{det}\left(\alpha_{i j}\right) \neq 0$, it follows from [1, p. 640] that $\operatorname{det} M(0)= \pm \operatorname{det}\left(\hat{\alpha}_{i j}\right) \neq 0$. Therefore, by [2, Theorem 1.2], we obtain $r\left(\wedge^{2} F\left(G^{\prime}\right)\right)$ $=k$.

LEMMA 3.6. If $r\left(F\left(G^{\prime}\right)\right)=2$ or 3 , then $H_{2}\left(F\left(G^{\prime}\right)\right)$ is not finitely generated over $Z$.

Proof. By [1, p. 640], we see that $\operatorname{det}\left(\hat{\alpha}_{i j}\right)=\left(\operatorname{det}\left(\alpha_{i j}\right)\right)^{n-1}$ for $n=2,3$. Thus we have det $M(0) \neq \pm 1,0$. Hence, by [15], $\wedge{ }^{2} F\left(G^{\prime}\right)$ is not finitely generated over $Z$.

LEMMA 3.7. Let $U=\left\langle a: t^{-1} a=2 a\right\rangle$ and $V$ be a finite $\Lambda$-module. If $V$ is $a$ $\Lambda$-homomorphic image of $U$, then $V$ is a cyclic group.

Proof. Let $\rho$ be a $\Lambda$-epimorphism of $U$ onto $V$, and $\mu$ the order of $\rho(a)$. Consider a quotient $W=U /\langle\mu a\rangle$. Then we have $W=\left\langle a: t^{-1} a=2 a, \mu a\right\rangle$ $=\left\langle a: t^{-1} a=2 a, \mu^{\prime} a\right\rangle$, where $\mu^{\prime}$ is an integer such that $\mu=2^{i} \mu^{\prime}$ and $\left(\mu^{\prime}, 2\right)=1$. Therefore $W$ is cyclic of order $\mu^{\prime}$. Since $V$ is a quotient of $W$, the proof is completed. 
4. Proof of Theorem. Let $n$ be the torsion-free rank of $G^{\prime}$. By Lemmas 3.3 and 3.4, we have

$$
r\left(G^{\prime}\right)=r \overline{\left(\tilde{F}\left(G^{\prime}\right)\right)} \geqslant r\left(H_{2}\left(G^{\prime}\right)\right)=r\left(H_{2}\left(F\left(G^{\prime}\right)\right)\right) .
$$

It follows from Lemma 3.5 that $n \geqslant n(n-1) / 2$. Hence, we see that $1 \leqslant n \leqslant 3$.

Suppose that $n=2$ or 3 . Let $\tilde{E}_{1}$ and $E_{1}$ be the order ideals of $\overline{\tilde{F}\left(G^{\prime}\right)}$ and $\wedge^{2} F\left(G^{\prime}\right)$, respectively. Then, from (3) and (4), as $\Lambda$-modules, $\overline{F\left(G^{\prime}\right)}$ and $\wedge^{2} F\left(G^{\prime}\right)$ have square presentation matrices, and we see that $\tilde{E}_{1}=\left(c_{0} t^{n}+\cdots+c_{n-1} t+1\right)$ and $E_{1}=\left(t^{k}+d_{k-1} t^{k-1}+\cdots+d_{0}\right)$, where $k=n(n-1) / 2$ and $c_{i}, d_{i} \in Z$, and $c_{0}, d_{0} \neq 0$. Since $\wedge^{2} F\left(G^{\prime}\right)$ is a $\Lambda$-homomorphic image of $\tilde{F}\left(G^{\prime}\right)$, the polynomial $c_{0} t^{n}+\cdots+1$ must be contained in the ideal $E_{1}$. Hence, there exists a polynomial $h(t)$ of $Z[t]$ such that $c_{0} t^{n}+\cdots+1=h(t)\left(t^{k}+\cdots+d_{0}\right)$. Therefore, $d_{0}$ must be \pm 1 . However, this contradicts Lemma 3.6. Thus we conclude that $r\left(G^{\prime}\right)=1$. It follows from [5] that $G^{\prime} \cong Z[1 / 2] \oplus T\left(G^{\prime}\right)$ and $\overline{\tilde{F}\left(G^{\prime}\right)}=\left\langle a: t^{-1} a=2 a\right\rangle$.

Next, we will consider the torsion part $T=T\left(G^{\prime}\right)$ of $G^{\prime}$. For each prime $p$, let $T_{p}$ be the $p$-primary component of $T$. Then, as an abelian group, we have

$$
H_{2}\left(G^{\prime}\right) \cong \wedge^{2} G^{\prime} \cong \wedge^{2} Z[1 / 2] \oplus(Z[1 / 2] \otimes T) \oplus \wedge^{2} T \cong \bigoplus_{p \neq 2} T_{p} \oplus \wedge^{2} T .
$$

If the $p$-rank $r_{p}(T) \geqslant 3$, then we get $r_{p}\left(H_{2}\left(G^{\prime}\right)\right) \geqslant r_{p}\left(\wedge^{2} T\right) \geqslant 3$. Furthermore, if $r_{p}(T)=2$, then we have $p \neq 2$ from Proposition 3.2 and [5]. Hence, we obtain $r_{p}\left(H_{2}\left(G^{\prime}\right)\right) \geqslant r_{p}\left(T_{p}\right)=2$. However, since $H_{2}\left(G^{\prime}\right)$ is a $\Lambda$-homomorphic image of $\tilde{F}\left(G^{\prime}\right)=\left\langle a: t^{-1} a=2 a\right\rangle$, these contradict Lemma 3.7. Thus we conclude that $r_{2}(T)$ $=0$ and $r_{p}(T) \leqslant 1$ for any prime $p(\neq 2)$. (Note that $r_{2}(T) \neq 1[5]$.) Therefore, $T$ is cyclic of odd order, and $G^{\prime}$ has a $\Lambda$-module presentation

$$
\langle a, b: t a=2 a+\beta b, t b=\gamma b, \lambda b=0\rangle,
$$

where $\lambda$ is an odd integer, and $\beta, \gamma \in Z$. Moreover, using the arguments of pairing in [10], we see that $\gamma$ must be -1 .

Now, the second exterior power $\wedge^{2} G^{\prime}$ is a cyclic group of order $\lambda$ generated by $a \wedge b$, and the action of $t$ on $\wedge^{2} G^{\prime}$ is given by $t(a \wedge b)=-2(a \wedge b)$. Let $\psi$ be the $\Lambda$-epimorphism of $\overline{\tilde{F}\left(G^{\prime}\right)}$ onto $\wedge^{2} G^{\prime}$ and let $\psi(a)=q(a \wedge b), 0 \leqslant q<\lambda$. Then, we have $t \cdot \psi(2 a)=t(2 q \cdot a \wedge b)=-4 q(a \wedge b)$. On the other hand, we get $t \cdot \psi(2 a)$ $=\psi(t \cdot 2 a)=\psi(a)=q(a \wedge b)$. Therefore, we obtain $5 q(a \wedge b)=0$, or $5 q \equiv 0$ $\bmod \lambda$. Furthermore, since $\psi$ is an epimorphism, it is clear that $q$ is relatively prime to $\lambda$. Thus we have $5 \equiv 0 \bmod \lambda$, i.e., $\lambda=1$ or 5 . The proof is completed.

REMARK. When $\lambda=1$, from (5) $G$ has a presentation

$$
\left\langle a, t: t^{-1} a t=a^{2}\right\rangle \text {. }
$$

Therefore, it can be realized as the group of a ribbon 2-knot in the 4-sphere $S^{4}[8]$. In the case $\lambda=5$, setting $a^{\prime}=a+2 \beta b$ in (5), we have a $\Lambda$-module presentation for $G^{\prime}$

$$
\left\langle a^{\prime}, b: t a^{\prime}=2 a^{\prime}, t b=-b, 5 b=0\right\rangle .
$$

Thus, we obtain (*) as a presentation for $G$. (It is easy to see that this group is an $n$-knot group, $n \geqslant 3$.) 


\section{REFERENCES}

1. N. Bourbaki, Elements of mathematics, Algebra. I, Addison-Wesley, Reading, Mass., 1973.

2. R. H. Crowell, The group $G^{\prime} / G^{\prime \prime}$ of a knot group $G$, Duke Math. J. 30 (1963), 349-354.

3. L. Fuchs, Infinite abelian groups, vols. 1 and 2, Academic Press, New York, 1970.

4. M. A. Gutierrez, An exact sequence calculation for the second homotopy of a knot, Proc. Amer. Math. Soc. 32 (1972), 571-577.

5. J. C. Hausmann and M. Kervaire, Sous-groupes dérivés des groupes de noeuds, Enseign. Math. 24 (1978), 111-123.

6. G. Higman, B. H. Neumann and H. Neumann, Embedding theorems for groups, J. London Math. Soc. 24 (1949), 247-254.

7. H. Hopf, Fundamentalgruppe und zweite Bettische gruppe, Comment. Math. Helv. 14 (1942), 257-309.

8. T. Kanenobu, Non-ribbon n-knots with Seifert manifolds homeomorphic to punctured $S^{n} \times S^{1}$, Math. Sem. Notes Kobe Univ. 10 (1982), 69-74.

9. M. Kervaire, Les noeuds de dimensions supérieures, Bull. Soc. Math. France 93 (1965), 225-271.

10. J. Levine, Knot modules. I, Trans. Amer. Math. Soc. 229 (1977), 1-50.

11. __ Some results on higher dimensional knot groups, Lecture Notes in Math., vol. 685, Springer-Verlag, Berlin and New York, 1978, pp. 243-269.

12. R. C. Lyndon and P. E. Schupp, Combinatorial group theory, Springer-Verlag, Berlin, 1977.

13. W. Magnus, A. Karrass and D. Solitar, Combinatorial group theory, Wiley, New York, 1966.

14. D. G. Northcott, $A$ first course of homological algebra, Cambridge Univ. Press, London and New York, 1973.

15. E. S. Rapaport, On the commutator subgroup of a knot group, Ann. of Math. (2) 71 (1960), 157-162.

16. D. J. S. Robinson, $A$ course in the theory of groups, Graduate Texts in Math., vol. 80, Springer-Verlag, Berlin, 1982.

Faculty of Science, Kwansei Gakuin University, Nishinomiya, Hyogo 662, Japan 